\title{
Global phase-locking in finite populations of phase-coupled oscillators
}

\author{
Mark Verwoerd and Oliver Mason \\ Hamilton Institute, National University of Ireland \\ \{mark. verwoerd, oliver.mason\}@nuim. ie
}

July 23, 2007

\begin{abstract}
We present new necessary and sufficient conditions for the existence of fixed points in a finite system of coupled phase oscillators on a complete graph. We use these conditions to derive bounds on the critical coupling.
\end{abstract}

\section{Introduction}

The phenomenon of synchronization arises in a wide variety of application areas across neuroscience, biology, engineering and physics $[6,17,2,16,5]$. As such, the identification and study of structures and mechanisms that support the onset of synchronized behaviour is a key issue in the theory of interconnected dynamical systems. In particular, there has been a great deal of interest across the mathematics, physics and engineering communities in the development and analysis of simple mathematical models of synchronization $[9,19,20,3,21,22]$.

To date, one of the most widely-studied frameworks for the analysis of synchronization is the so-called Kuramoto model of phase coupled oscillators $[10,23]$. In fact, this model has been used in numerous applications in the chemical and biological sciences, and its basic properties have been analysed using a combination of numerical and analytical techniques [11, 23, 24, 1]. The basic Kuramoto model is comprised of a system of coupled oscillators, which may have different natural frequencies, where the coupling between two oscillators is given by a weighted sinusoidal function of the difference of their phases. The weights used in the model are typically taken to be the same for all pairs of oscillators and are given by the ratio of a fixed parameter, the coupling strength, to the network size.

The aspect of the Kuramoto model that has attracted most attention to date is the manner in which the onset of synchronization depends on the strength of coupling between the oscillators. For instance, at very low values of the 
coupling strength, little or no synchronization is observed. As the coupling strength is increased, some partial synchronization appears in the network up to a threshold value of the coupling strength, referred to here as the critical coupling, at which fully synchronized behaviour emerges $[9,3]$. The mechanism of (de)synchronization in finite populations of oscillators has been described in considerable detail $[13,12]$. In particular, when the coupling strength drops below its critical value and as it continues to decrease, the system undergoes a series of so called frequency-splitting bifurcations. At each such bifurcation, the ensemble of oscillators subdivides into smaller and smaller groups of oscillators with identical average frequency, until eventually all oscillators oscillate at their own intrinsic frequency. A detailed analysis of this behaviour for a system with three oscillators was given in [13]. While the aforementioned contributions focus on the behaviour of the system in the subcritical coupling regime, the present paper studies globally phase-locked solutions, which by definition only exist in the supercritical coupling regime.

Another aspect of the Kuramoto model to have attracted attention recently is the emergence of phase chaos $[14,18]$ in systems of dimension four and higher. A generic feature of coupled oscillator systems, phase chaos in the Kuramoto model is most prominent in systems with relatively low dimension (comprising between ten and fifteen oscillators) [14]. Again this phenomenon can only exist in the subcritical coupling regime, and we shall not further consider it here.

In the original Kuramoto model, it is assumed that all pairs of oscillators in the network are connected with the same coupling strength [10]. This type of coupling is referred to as 'all-to-all' coupling and corresponds to a network in which the underlying graph is complete [4]. Extensions of the Kuramoto model to lattices [8] and rings [20] have also been considered, and more recently the dynamics of coupled oscillators on networks with small-world [27, 7] and scale-free [15] topologies have started to attract a lot of interest. More generally, there are many fundamental questions relating to the interplay between a network's topology and dynamical processes taking place on it which are still unanswered. The work described in [6], which proposes an extension of group-based symmetry, using the so-called groupoid formalism, as a means of classifying possible behaviours for networked dynamical systems, is particularly noteworthy in this context.

Many of the recent results concerned with the dynamics and synchronization of coupled oscillators have either been based on numerical simulations or else have been derived for the limiting case of networks of infinite size. In contrast, relatively few rigorous results are available for finite-size networks [23, 9]. In this paper, we shall be concerned with synchronization in finite systems of coupled oscillators. Specifically: we shall establish (new) necessary and sufficient conditions for the existence of fixed points in a finite system of coupled oscillators (see also $[25,26]$ ); compute bounds on the critical coupling strength for such systems; and provide insights into the number of fixed points possible under strong coupling. Our analysis is in the spirit of the work presented in $[9,3]$, and places particular emphasis on the existence of fixed points. Of course, the stability of such fixed points is also a topic of great interest, and has been con- 
sidered in $[9,19,20,3]$. However, we shall not explicitly address the question of stability in the current paper.

The outline of the paper is as follows. In Section 2, we introduce the $\mathrm{Ku}$ ramoto model, and review some of its basic properties. Here, we also give a formal definition of the critical coupling, which is essentially the lowest value of the coupling strength for which fixed points exist. In Section 3, we show that fixed points will always exist for sufficiently strong coupling (essentially proving that the critical coupling is a finite number), and then, in Section 4 provide lower bounds on the critical coupling. Section 5 contains necessary and sufficient conditions for the existence of fixed points, which are then used in Section 6 to describe an algorithm for computing the critical coupling. Section 7 contains a numerical example to illustrate the results of the paper and finally, in Section 8 we present our concluding remarks.

\section{Mathematical Preliminaries and The Kuramoto model}

\subsection{Basic Notation}

Throughout the paper, $\mathbb{R}(\mathbb{C})$ denotes the field of real (complex) numbers, $\mathbb{R}^{N}$ $\left(\mathbb{C}^{N}\right)$ denotes the vector space of all $N$-tuples of real (complex) numbers, and $\mathbb{R}^{N \times N}\left(\mathbb{C}^{N \times N}\right)$ denotes the space of $N \times N$ matrices with entries in $\mathbb{R}(\mathbb{C}) . i$ is used to denote the complex number satisfying $i^{2}=-1$. For a vector $x \in \mathbb{R}^{N}$, $x_{i}$ denotes the $i^{\text {th }}$ entry of $x$. Also, $\mathbf{1}_{N}$ denotes the vector in $\mathbb{R}^{N}$, all of whose entries are equal to one.

We shall use $V$ to denote the projection matrix in $\mathbb{R}^{N \times N}$ given by

$$
\left[V_{i j}\right]:=\left\{\begin{array}{cc}
\frac{N-1}{N} & j=i \\
-\frac{1}{N} & j \neq i
\end{array}, \quad i, j=1, \ldots, N\right.
$$

and $V \mathbb{R}^{N}$ shall denote the image of $\mathbb{R}^{N}$ under $V$. Formally,

$$
V \mathbb{R}^{N}:=\left\{x \in \mathbb{R}^{N}: \sum_{j=1}^{N} x_{j}=0\right\} .
$$

\subsection{The Basic Kuramoto Model}

The basic Kuramoto model of phase-coupled oscillators under the assumption of all-to-all coupling is given by

$$
\dot{\theta}_{i}=\omega_{i}+\frac{k}{N} \sum_{j=1}^{N} \sin \left(\theta_{j}-\theta_{i}\right), \quad i=1, \ldots, N .
$$

Here, $\theta_{i}(\cdot) \in \mathbb{R}\left(S^{1}\right)$ and $\omega_{i} \in \mathbb{R}$ respectively denote the phase and intrinsic (or natural) frequency of oscillator $i$, and the constant $k \in \mathbb{R}_{+}$is a global coupling coefficient. 
This model can be described more compactly in vector notation as

$$
\dot{\theta}=\omega+k f(\theta)
$$

where $\theta(t):=\left(\theta_{1}(t), \ldots, \theta_{N}(t)\right), \omega:=\left(\omega_{1}, \ldots, \omega_{N}\right)$, and the mapping $f: \mathbb{R}^{N} \mapsto$ $\mathbb{R}^{N}$ is given by

$$
\begin{aligned}
f(\xi) & =\left(f_{1}(\xi), \ldots, f_{N}(\xi)\right) \\
f_{i}(\xi) & :=\frac{1}{N} \sum_{j=1}^{N} \sin \left(\xi_{j}-\xi_{i}\right) \quad 1 \leq i \leq N
\end{aligned}
$$

The assumption of all-to-all coupling is naturally very restrictive, and ought to be relaxed in order for this work to be more directly applicable to the modelling of biological systems, or most engineering systems for that matter. Work towards this end is underway and we hope to be able to present some results in the near future. Meanwhile, in this paper, we shall focus exclusively on configurations with all-to-all coupling. First of all, we recall some fundamental notions in the theory of synchronized oscillators.

\subsection{The order parameter}

Let $\mathbb{D}$ denote the complex unit disc $\{z \in \mathbb{C}:|z| \leq 1\}$. Then define $r: \mathbb{R}^{N} \mapsto \mathbb{D}$, by:

$$
r(\xi):=\frac{1}{N} \sum_{j=1}^{N} e^{i \xi_{j}}
$$

Let $r^{-1}(z):=\left\{\xi \in \mathbb{R}^{N}: r(\xi)=z\right\}$ denote the preimage of $r$, and note that the preimage is nonempty for all $z \in \mathbb{D}$ provided $N \geq 2$. We introduce the notation $\mathcal{R}_{0}:=r^{-1}(0)$. Then, for $\xi \in \mathbb{R}^{N}$, we may express $r(\xi)$ in polar coordinates:

$$
r(\xi)= \begin{cases}R(\xi) e^{i \psi(\xi)} & \xi \in \mathbb{R}^{N} \backslash \mathcal{R}_{0} \\ 0 & \xi \in \mathcal{R}_{0}\end{cases}
$$

Here, $R: \mathbb{R}^{N} \mapsto[0,1]$ and $\psi: \mathbb{R}^{N} \backslash \mathcal{R}_{0} \mapsto[0,2 \pi)$ are respectively defined as

$$
R(\xi):=\sqrt{\left(\frac{1}{N} \sum_{j=1}^{N} \sin \left(\xi_{j}\right)\right)^{2}+\left(\frac{1}{N} \sum_{j=1}^{N} \cos \left(\xi_{j}\right)\right)^{2}},
$$

and

$$
\psi(\xi):=\arctan \left(\frac{\frac{1}{N} \sum_{j=1}^{N} \sin \left(\xi_{j}\right)}{\frac{1}{N} \sum_{j=1}^{N} \cos \left(\xi_{j}\right)}\right)
$$




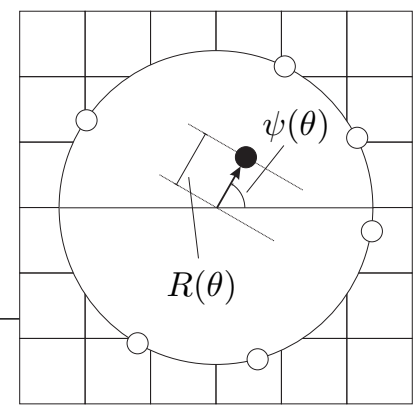

Figure 1: The order parameter $r(\theta):=R(\theta) e^{i \psi(\theta)}$ is defined as the centroid (closed circle) of the set of unit vectors (open circles) associated with the phases of the oscillators.

The following properties of the maps $R(\cdot)$ and $\psi(\cdot)$ follow immediately from Eqn. (5):

$$
\begin{gathered}
R\left(\xi+c \mathbf{1}_{N}\right):=\left|\frac{1}{N} \sum_{j=1}^{N} e^{i\left(\xi_{j}+c\right)}\right|=\left|e^{i c}\right| R(\xi)=R(\xi) \quad \forall \xi \in \mathbb{R}^{N} ; \\
\psi\left(\xi+c \mathbf{1}_{N}\right)=\psi(\xi)+c \quad \bmod 2 \pi \quad \forall \xi \in \mathbb{R}^{N} \backslash \mathcal{R}_{0} .
\end{gathered}
$$

In the physics literature, $r(\cdot)$ is known as the order parameter, and is used to characterize the amount of order or synchronization in the system (2). The idea is to think of the phase $\theta_{j}$ of oscillator $j$ as a unit vector $e^{i \theta_{j}}$ in $\mathbb{C}$; the order parameter then corresponds to the geometric centroid of the set of vectors $\left\{e^{i \theta_{j}}: j=1, \ldots, N\right\}$, as illustrated in Figure 1. The magnitude of the order parameter, given by $R(\theta)$, serves as a measure of the order in the system, in the sense that the closer the vectors are to being perfectly aligned, the closer $R(\theta)$ is to its maximal value 1 , while vectors that are far from alignment will give rise to values of $R(\theta)$ significantly smaller than 1 .

It follows from (6) that for $\xi \in \mathbb{R}^{N} \backslash \mathcal{R}_{0}$,

$$
\begin{aligned}
R(\xi) & =e^{-i \psi(\xi)} r(\xi) \\
& =\frac{1}{N} \sum_{j=1}^{N} e^{i\left(\xi_{j}-\psi(\xi)\right)} .
\end{aligned}
$$

Equating real and imaginary parts in (11), we immediately see that for $\xi \in$ $\mathbb{R}^{N} \backslash \mathcal{R}_{0}$ :

$$
\begin{gathered}
R(\xi)=\frac{1}{N} \sum_{j=1}^{N} \cos \left(\psi(\xi)-\xi_{j}\right) ; \\
\sum_{j=1}^{N} \sin \left(\psi(\xi)-\xi_{j}\right)=0 .
\end{gathered}
$$


Both of these identities shall prove useful throughout the paper.

Before proceeding, note that the function $f: \mathbb{R}^{N} \mapsto \mathbb{R}^{N}$ given by (4) can be written in terms of the functions $R(\cdot)$ and $\psi(\cdot)$ as

$$
f_{i}(\xi):=\left\{\begin{array}{ll}
R(\xi) \sin \left(\psi(\xi)-\xi_{i}\right) & \xi \in \mathbb{R}^{N} \backslash \mathcal{R}_{0} \\
0 & \xi \in \mathcal{R}_{0}
\end{array},\right.
$$

for $1 \leq i \leq N$.

\subsection{Fixed points and global phase-locking}

Let $\langle\omega\rangle$ denote the sample mean of the natural frequencies, $\langle\omega\rangle:=\frac{1}{N} \sum_{j=1}^{N} \omega_{j}$. Similarly, let $\langle\theta(t)\rangle$ denote the mean phase of a solution of (2) at time $t$. In general, $\langle\omega\rangle$ and $\langle\theta(t)\rangle$ will be non-zero. However, we shall now show that for the study of phase-locked solutions of (2), we may assume without loss of generality that $\langle\omega\rangle=0,\langle\theta(t)\rangle=0$ for $t \geq t_{0}$. This helps to simplify the analysis of phase-locked solutions of (2), as it allows us to transform the problem into a question of fixed point existence for a lower-dimensional system.

Consider the new coordinates

$$
x_{i}(t):=\theta_{i}(t)-\langle\theta(t)\rangle, \quad i=1, \ldots, N .
$$

Then $x(t):=V \theta(t)$. Similarly, define $\Omega:=V \omega$. In the new coordinates, the system dynamics are given by:

$$
\dot{x}=\Omega+k f(x), \quad x(t) \in V \mathbb{R}^{N},
$$

where $f(\cdot)$ is defined in (4).

The key point here is that as $\langle\Omega\rangle=0, V \mathbb{R}^{N}$ is invariant under (17). To avoid confusion, we shall use $x(t)$ to denote solutions to the system (17) on $V \mathbb{R}^{N}$, while $\theta(t)$ shall be used to denote solutions to the original Kuramoto system (2) on $\mathbb{R}^{N}$. Our main concern for the remainder of the paper is to find conditions on $k$ and $\Omega$ under which the system (17) has one or more fixed points in the sense of the following definition.

Definition 1 (fixed point) Given $\omega \in \mathbb{R}^{N}$, let $\Omega:=V \omega$. We say that $x \in$ $V \mathbb{R}^{N}$ is a fixed point (of the system (17)) if

$$
k f(x)=-\Omega .
$$

There is a natural correspondence between fixed points of (17) and phaselocked solutions of (2). In fact, for every fixed point $x^{*} \in V \mathbb{R}^{N}$ there is a 1-dimensional manifold $\mathcal{M}:=\left\{\theta \in \mathbb{R}^{N}: \theta=x^{*}+\langle\omega\rangle t, t \in \mathbb{R}\right\}$ that is invariant under the original system dynamics (2). More precisely, let $x^{*}$ be a fixed point and let $\theta^{0} \in \mathbb{R}^{N}$ be such that $V \theta^{0}=x^{*}$. Then the solution $\theta(t)$ of the system (2) with initial condition $\theta\left(t_{0}\right)=\theta^{0}$ satisfies

$$
\theta_{i}(t)-\theta_{j}(t)=\theta_{i}^{0}-\theta_{j}^{0}
$$


for all $t \geq t_{0}$ and all $(i, j)$. In other words, a fixed point in the sense of Definition 1 corresponds to a situation in which each oscillator is phase-locked to every other and moves at constant speed $\dot{\theta}_{i}=\langle\omega\rangle$. We shall refer to this phenomenon as global phase-locking. In the literature, it is also known as full (or complete) synchronization. See also [9].

\subsection{Critical coupling}

We next define the notion of critical coupling, which is central to the work of the rest of the paper. Essentially, the critical coupling is the smallest $k$ for which the system (17) has at least one fixed point. Formally, we have the following definition.

Definition 2 Given $\omega \in \mathbb{R}^{N}$, let $\Omega:=V \omega$. We define the critical coupling, $k_{c}$, as follows:

$$
k_{c}:=\inf _{k}\left\{k \in \mathbb{R}_{+}: \exists x \in V \mathbb{R}^{N} \text { s.t. } k f(x)=-\Omega\right\} .
$$

Note that this definition of the critical coupling, which is equivalent to that of $K_{L}$ in [9], does not coincide with the traditional notion used in the physics literature. Indeed, the traditional notion of critical coupling is defined in terms of the lowest value of $k$ for which there exists at least one solution $x(t), t \geq$ $t_{0}$, and a constant $c \in(0,1]$ such that $R(x(t))=c$ for all $t \geq t_{0}$ (so called stationary or steady solutions [23]). Note that these solutions are not necessarily fixed points, although, in finite dimensions, the probability of finding stationary solutions that are not fixed points is vanishingly small. In his original analysis, Kuramoto showed that in the limiting case when $N$ tends to infinity, stationary solutions always exist for large enough $k$, provided the distribution of natural frequencies is symmetric. Our definition, although more restrictive in a sense, does not impose any restriction on the shape of the distribution of natural frequencies other than that it should have compact support. In fact, it follows from the result of Lemma 4 below that, if the distribution of natural frequencies does not have compact support, then the critical coupling will exceed any finite number with probability tending to 1 as $N$ tends to infinity. In this paper we shall therefore focus on distributions with compact support.

\section{Existence of fixed points under strong cou- pling}

In this section we shall show that, provided the distribution of intrinsic frequencies has compact support, the critical coupling given in Definition 2 is always finite. Following on from this, in the next section, we shall derive a number of lower bounds for the value of the critical coupling. There are two steps in the derivation given here: first, we characterize the fixed points of the homogeneous system

$$
\dot{x}=k f(x) \text {. }
$$


Then, in the second step, we use a perturbation argument to show that for every fixed point of the homogeneous system, we can find an open set containing it, such that, under strong enough coupling, the original system (17) has a unique fixed point on this set. As a first step, the following lemma characterises the fixed points of the homogeneous system.

Lemma 1 Let $f(\cdot)$ be given by (4) and $\xi \in \mathbb{R}^{N}$. We have that $f(\xi)=0$ if and only if one or both of the following conditions are satisfied

(a) $R(\xi)=0$;

(b) $\sin \left(\xi_{i}-\xi_{j}\right)=0$ for all $(i, j)$.

Proof: Sufficiency of conditions $(a)$ and $(b)$ follows from (15) and (4) respectively. To prove necessity, suppose $f(\xi)=0$ and $R(\xi) \neq 0$ (if $R(\xi)=0$ we are done). It follows that $\sin \left(\psi(\xi)-\xi_{i}\right)=0$ for all $i$. This implies that there exist integers $k_{i} \in \mathbb{Z}, i=1,2, \ldots N$ such that $\psi(\xi)-\xi_{i}=k_{i} \pi$ for all $i$, and we have that $\xi_{i}-\xi_{j}=\left(k_{j}-k_{i}\right) \pi$. We conclude that $\sin \left(\xi_{i}-\xi_{j}\right)=0$ for all $(i, j)$.

Remark 1 It is not hard to see that conditions (a) and (b) in Lemma 1 are mutually exclusive if and only if the dimension $N$ is odd. We shall prove necessity. Suppose conditions $(a)$ and (b) both hold and suppose furthermore that $N$ is odd. Then for all $(i, j)$ we have that either $\cos \left(\xi_{i}-\xi_{j}\right)=1$ or $\cos \left(\xi_{i}-\xi_{j}\right)=-1$. We write $R^{2}(\xi)=\frac{1}{N^{2}} \sum_{i, j} \cos \left(\xi_{i}-\xi_{j}\right)=\frac{1}{N^{2}}\left(N+2 \sum_{i, j>i} \cos \left(\xi_{i}-\xi_{j}\right)\right)$. Since $R(\xi)=0$ by assumption, it follows that $2 \sum_{i, j>i} \cos \left(\xi_{i}-\xi_{j}\right)=-N$. The left hand side evaluates to an even integer. By assumption, the number on the right hand side is odd. We arrive at a contradiction and conclude that if $N$ is odd, conditions (a) and (b) cannot both hold.

Next we shall prove that the fixed points of our $N$-dimensional system (17) can be found by solving a system of $N-1$ equations in as many variables. We have the following result:

Lemma 2 Let $p \in\{1, \ldots, N\}$ and let $x^{*} \in V \mathbb{R}^{N}$. Then $x^{*}$ is a fixed point of (17) if and only if $k f_{i}\left(x^{*}\right)=-\Omega_{i}$ for $i \neq p$.

Proof: The proof of necessity is trivial. To prove sufficiency, recall that

$$
\sum_{j=1}^{N}\left(\Omega_{j}+k f_{j}(x)\right)=0 \quad \text { for all } x \in \mathbb{R}^{N} .
$$

Now suppose $k f_{i}(x)=-\Omega_{i}$ for all $i \neq p$. Then it follows from (22) that $\Omega_{p}+k f_{p}(x)=0$. In other words, it follows that $k f_{i}(x)=-\Omega_{i}$ for all $i$. We conclude that $x$ is a fixed point. 
Let $x^{*} \in V \mathbb{R}^{N}$ be a fixed point of the homogeneous system (21) such that $R\left(x^{*}\right) \neq 0$. We shall now show that locally, in a neighborhood of $x^{*}$, the system of equations

$$
\left\{\begin{array}{ccc}
-\Omega_{1} & = & k f_{1}\left(x_{1}, \ldots, x_{N-1},-\sum_{j=1}^{N-1} x_{j}\right) \\
\vdots & & \vdots \\
-\Omega_{N-1} & = & k f_{N-1}\left(x_{1}, \ldots, x_{N-1},-\sum_{j=1}^{N-1} x_{j}\right)
\end{array}\right.
$$

has a unique solution, provided $k$ is large enough. It follows directly from Lemma 2 that every solution of (23) defines a fixed point and, conversely, that every fixed point satisfies (23). We proceed as follows. Let $x^{*} \in V \mathbb{R}^{N}$. We define the Jacobian $J\left(x^{*}\right)$, as follows:

$$
\left[J_{i j}\left(x^{*}\right)\right]:=\left.\frac{\partial f_{i}\left(x_{1}, \ldots,-\sum_{j=1}^{N-1} x_{j}\right)}{\partial x_{j}}\right|_{x=x^{*}},
$$

where $i, j=1, \ldots, N-1$. We have the following result:

Lemma 3 Let $f(\cdot)$ be given by (4) and suppose that $x^{*} \in V \mathbb{R}^{N}$ satisfies $f(x)=$ 0 and $R(x) \neq 0$. Then $\operatorname{det}\left(J\left(x^{*}\right)\right) \neq 0$.

Proof: Let $x^{*}$ be a fixed point of the homogeneous system and suppose $R\left(x^{*}\right) \neq 0$. Then by Lemma 1 , we have that $\sin \left(x_{j}^{*}-x_{i}^{*}\right)=0$ for all $(i, j)$, and it follows that

$$
\begin{aligned}
\cos \left(x_{j}^{*}-x_{i}^{*}\right) & =\cos \left(\left(x_{j}^{*}-x_{s}^{*}\right)-\left(x_{i}^{*}-x_{s}^{*}\right)\right) \\
& =\cos \left(x_{j}^{*}-x_{s}^{*}\right) \cos \left(x_{i}^{*}-x_{s}^{*}\right)
\end{aligned}
$$

for all $s$ and all $(i, j)$. The claim is that $J\left(x^{*}\right)$ is nonsingular. To prove this, we proceed as follows. From the definition, we have that

$$
J_{i j}\left(x^{*}\right)=\left\{\begin{array}{ll}
-\sum_{m=1, m \neq i}^{N-1} \cos \left(x_{m}^{*}-x_{i}^{*}\right)-2 \cos \left(x_{N}^{*}-x_{i}^{*}\right) & i=j \\
\cos \left(x_{j}^{*}-x_{i}^{*}\right)-\cos \left(x_{N}^{*}-x_{i}^{*}\right) & i \neq j
\end{array} .\right.
$$

Using the aforementioned identity, setting $s=N$, we rewrite (26), as follows,

$$
J_{i j}\left(x^{*}\right)= \begin{cases}-\left(\sum_{m=1, m \neq i}^{N-1} \cos \left(x_{m}^{*}-x_{N}^{*}\right)+2\right) \cos \left(x_{i}^{*}-x_{N}^{*}\right) & i=j \\ \left(\cos \left(x_{j}^{*}-x_{N}^{*}\right)-1\right) \cos \left(x_{i}^{*}-x_{N}^{*}\right) & i \neq j\end{cases}
$$

Inspection shows that the rank of $J\left(x^{*}\right)$ is invariant under permutations of the components of $x^{*}$. Hence we can assume, without loss of generality, that there exists $\rho \in\{0, \ldots, N-1\}$, such that

$$
\cos \left(x_{j}^{*}-x_{N}^{*}\right)= \begin{cases}-1, & 1 \leq i \leq \rho \\ +1, & \rho+1 \leq i \leq N\end{cases}
$$


Under this assumption $J\left(x^{*}\right)$ takes the form

$$
J\left(x^{*}\right)=\left(\begin{array}{ll}
A & 0 \\
C & B
\end{array}\right)
$$

where $A$ and $B$ are square matrices of dimension $\rho \times \rho$ and $(N-1-\rho) \times(N-1-\rho)$ respectively. It follows that $J\left(x^{*}\right)$ is nonsingular if and only if $A$ and $B$ are nonsingular. Inspection shows that $A=(N-2 \rho) I+2 \mathbf{1 1}{ }^{T}$ and $B=(2 \rho-N) I$. It follows that $A$ or $B$ is singular if and only if $N=2 \rho$. In case $N$ is odd, this condition is never satisfied. In case $N$ is even this condition, combined with (25) and the fact that $R^{2}(x)=\frac{1}{N^{2}} \sum_{i, j} \cos \left(x_{i}-x_{j}\right)$, implies that $R\left(x^{*}\right)=0$, which contradicts our starting assumption. We conclude that, under the hypotheses of the lemma, $J$ is nonsingular. This concludes the proof.

Let $\Pi: \mathbb{R}^{N-1} \mapsto V \mathbb{R}^{N}$, be given as

$$
(\Pi(y))_{i}:= \begin{cases}y_{i} & \text { for } i=1,2, \ldots N-1 \\ -\sum_{j=1}^{N-1} y_{j} & \text { for } i=N .\end{cases}
$$

and note that $\Pi$ has an inverse $\Pi^{-1}$ that is defined everywhere in $V \mathbb{R}^{N}$. We are now ready to state the main result:

Theorem 1 Let $f(\cdot)$ be given by (4) and $x^{*} \in V \mathbb{R}^{N}$ be such that $f\left(x^{*}\right)=0$ and $R\left(x^{*}\right) \neq 0$. Also, let $\Omega \in V \mathbb{R}^{N}$. Then there exists $K \in \mathbb{R}$, and an open set $U \in \mathbb{R}^{N-1}$ such that (a) $\Pi^{-1}\left(x^{*}\right)$ is an interior point of $U$; and (b) for all $k>K$, the system of equations (23) has a unique solution on $U$.

Proof: $\quad$ Define $y^{*}:=\Pi^{-1}\left(x^{*}\right)$, and let $g: \mathbb{R}^{N-1} \mapsto \mathbb{R}^{N-1}$ be given as $g_{i}(y):=f_{i}\left(y_{1}, \ldots, y_{N-1},-\sum_{j=1}^{N-1} y_{j}\right), i=1, \ldots, N-1$. Note that $g\left(y^{*}\right)=0$. Also, by Lemma 3 , we have that $\operatorname{det}\left(\frac{\partial g}{\partial y}\left(y^{*}\right)\right) \mid \neq 0$. Under these conditions, the Inverse Function Theorem says that there exists an open set $U \subset \mathbb{R}^{N-1}$ containing $y^{*}$ such that $\left.g\right|_{U}: U \mapsto g(U)$ is a diffeomorphism. By continuity (and bijectivity) of $g^{-1}$ there exists $\delta>0$ such that for all $z \in \mathbb{R}^{N-1}$ satisfying $\|z\|<\delta$, the equation $g(y)=z$ has a unique solution on $U$. Now let $z$ be given as $z_{i}:=-\Omega_{i} / k$. Since, by assumption, $\max _{i}\left|\Omega_{i}\right|<\infty$, it follows that, provided $k$ is large enough, the system of equations $\left\{k g_{i}(y)=-\Omega: i=1, \ldots, N-1\right\}$ has a unique solution on $U$. This concludes the proof.

As alluded to earlier, there is a unique correspondence between solutions of (23) and the fixed points of the system (17). Indeed, by Lemma 2 we have that if $y$ is a solution of $(23)$, then $\Pi^{-1}(y)$ is a fixed point, and conversely, if $y$ is a fixed point, then $\Pi(y)$ is a solution of (23). Thus, an immediate consequence of Theorem 1 is that for large enough $k$, the system (23) will have at least one fixed point. In other words, Theorem 1 tells us that the critical coupling, $k_{\mathrm{c}}$, is always finite.

Note furthermore that the proof of Theorem 1 does not require detailed knowledge of the coupling function $g$ and that, as such, its applicability is not restricted to networks with all-to-all coupling. To illustrate this, consider the 
case of a 4-cycle, where $g$ is given as

$$
\begin{aligned}
& g_{1}(y)=\frac{1}{4} \sin \left(y_{2}-y_{1}\right)+\frac{1}{4} \sin \left(-2 y_{1}-y_{2}-y_{3}\right) \\
& g_{2}(y)=\frac{1}{4} \sin \left(y_{3}-y_{2}\right)+\frac{1}{4} \sin \left(y_{1}-y_{2}\right) \\
& g_{3}(y)=\frac{1}{4} \sin \left(-2 y_{3}-y_{1}-y_{2}\right)+\frac{1}{4} \sin \left(y_{2}-y_{3}\right) .
\end{aligned}
$$

We have that $g(0)=0$ and $\operatorname{det}\left(\frac{\partial g}{\partial y}(0)\right)=-\frac{1}{4} \neq 0$. This implies that for $k$ large enough, the system of equations $\left\{k g_{i}(y)=-\Omega_{i}: i=1,2,3\right\}$ has a unique solution on some open set containing the origin.

Lastly, note that continuity of $g^{-1}$ implies that the fixed points of the original system (17) will converge to the fixed points of the homogeneous system (21) as $k$ tends to infinity.

\section{Lower bounds on the critical coupling}

In the previous section we showed that the critical coupling is finite, provided the oscillator's intrinsic frequencies are finite. In the present section we shall investigate in more detail the relation between the distribution of intrinsic frequencies and the critical coupling. In particular, we shall derive various lower bounds and discuss some of these bound's implications for the system's dynamic behaviour.

First, let us observe that $k_{\mathrm{c}}$ (Definition 2) is lower bounded by the $l^{\infty}$ norm of $\Omega$ :

$$
k_{\mathrm{c}} \geq\|\Omega\|_{\infty}:=\max _{i}\left|\omega_{i}-\langle\omega\rangle\right| .
$$

This follows trivially from inspection of Eqn. (17). In order to derive another lower bound, we shall need the following result:

Lemma 4 Let $f(\cdot)$ be given by (4). Then:

1. For all $x \in \mathbb{R}^{N}$,

$$
\|f(x)\|_{2} \leq \sqrt{N R^{2}(x)\left(1-R^{2}(x)\right)}
$$

2. If $N$ is even, then for every $c \in[0,1]$ there exists $x \in V \mathbb{R}^{N}$ such that $R(x)=c$ and $\|f(x)\|_{2}=\sqrt{N R^{2}(x)\left(1-R^{2}(x)\right)} ;$

3. If $N$ is odd, then inequality (33) is strict for all $x \in \mathbb{R}^{N}$ such that $0<$ $R(x)<1$.

Proof: Part 1. Observe that inequality (33) is trivially satisfied when 
$x \in \mathcal{R}_{0}$. Suppose therefore that $x \in \mathbb{R}^{N} \backslash \mathcal{R}_{0}$. Then by definition

$$
\begin{aligned}
\|f(x)\|_{2}^{2} & :=\sum_{j=1}^{N}\left(f_{j}(x)\right)^{2} \\
& =R^{2}(x) \sum_{j=1}^{N} \sin ^{2}\left(\psi(x)-x_{j}\right),
\end{aligned}
$$

where $\psi(x)$ and $R(x)$ are the phase and magnitude of the order parameter, previously defined in (8) and (7) respectively. Introducing the shorthand notation $z_{i}(x):=\cos \left(\psi(x)-x_{i}\right)$, and using (13) we now rewrite (34), as follows:

$$
\|f(x)\|_{2}^{2}=\left(\frac{1}{N} \sum_{j=1}^{N} z_{j}(x)\right)^{2} \sum_{j=1}^{N}\left(1-z_{j}(x)^{2}\right) .
$$

To derive the desired inequality we pick a $c \in[0,1]$ and maximize $\|f(x)\|_{2}$ over the set $\left\{x \in \mathbb{R}^{N}: R(x)=c\right\}$. We shall not solve this optimization problem directly, but take an indirect route by considering another, easier optimization problem, whose solution will then give us an upper bound on the solution to the first problem. Then we shall show that, under certain conditions, the two solutions coincide.

To this end, let $c \in(0,1]$ and consider the constrained optimization problem

$$
\text { OPT 1: } \quad \begin{array}{ll}
\text { maximize } & \sum_{j=1}^{N}\left(1-z_{j}(x)^{2}\right) \\
\text { subject to } & \frac{1}{N} \sum_{j=1}^{N} z_{j}(x)=c, \quad x \in \mathbb{R}^{N} \backslash \mathcal{R}_{0}
\end{array}
$$

Note that the constraint is feasible for all values of $c$ in the specified interval. We shall denote the solution to OPT 1 as $s_{1}(c)$. Next consider a second optimization problem,

$$
\begin{array}{lll}
\text { OPT 2: } & \text { maximize } & \sum_{j=1}^{N}\left(1-y_{j}^{2}\right) \\
\text { subject to } & \frac{1}{N} \sum_{j=1}^{N} y_{j}=c, \quad y \in \mathbb{R}^{N} .
\end{array}
$$

and let the solution to this problem be denoted as $s_{2}(c)$. We then have that $s_{2}(c) \geq s_{1}(c)$ for all $c \in(0,1]$. In other words, the solution to OPT 1 is upper bounded by the solution to OPT 2 . The solution to OPT 2 can be found by means of standard Lagrange multiplier techniques. The optimum $s_{2}(c)=N\left(1-c^{2}\right)$, is attained when $y_{i}=c$ for all $i$. We conclude that

$$
\max _{\left\{x \in \mathbb{R}^{N}: R(x)=c\right\}}\|f(x)\|_{2}^{2} \leq N c^{2}\left(1-c^{2}\right),
$$

and hence,

$$
\|f(x)\|_{2} \leq \sqrt{N} R(x) \sqrt{1-R^{2}(x)} .
$$


for all $x \in \mathbb{R}^{N}$.

Part 2. To prove the second part of the theorem, let $c \in(0,1]$ and note that $s_{1}(c)=s_{2}(c)$ if and only there exists $x \in \mathbb{R}^{N} \backslash \mathcal{R}_{0}$ such that

$$
\cos \left(\psi(x)-x_{i}\right)=c
$$

for all $i$. Suppose $N$ is even and let $x$ be given as

$$
x_{i}:= \begin{cases}\arccos (c) & i=1, \ldots, \frac{N}{2} \\ -\arccos (c) & i=\frac{N}{2}, \ldots, N .\end{cases}
$$

Then $\sum_{j=1}^{N} x_{j}=0$, and, by definition, $x \in V \mathbb{R}^{N}$. Moreover, $\psi(x)=0$, and $\cos \left(\psi(x)-x_{i}\right)=c$ for all $i$. This completes the second part.

Part 3. To prove the third part, let $N$ be odd and suppose there exists $x \in \mathbb{R}^{N}$ such that Condition (38) is satisfied. Then it follows from the identity $\sin ^{2}\left(\psi(x)-x_{i}\right)+\cos ^{2}\left(\psi(x)-x_{i}\right)=1$ that there must exist $a \in\{-1,1\}^{N}$ such that $\sin \left(\psi(x)-x_{i}\right)=a_{i} \sqrt{1-c^{2}}$ for all $i$. By Identity (14), we have that $\sum_{j} \sin \left(\psi(x)-x_{j}\right)=0$, which, assuming $c \neq 1$, implies that $\sum_{j=1}^{N} a_{j}=0$. But this cannot be true unless $N$ is even. Thus we arrive at a contradiction and we conclude that if $N$ is odd then $s_{2}(c)>s_{1}(c)$ for all $c$ such that $0<c<1$. This concludes the proof.

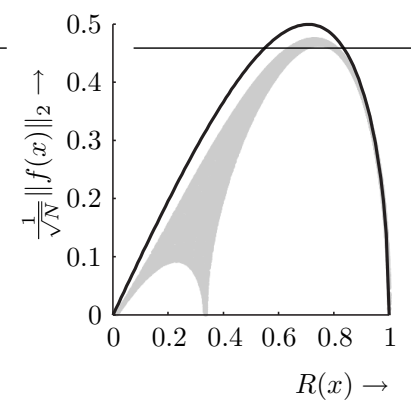

(a) $N=3$

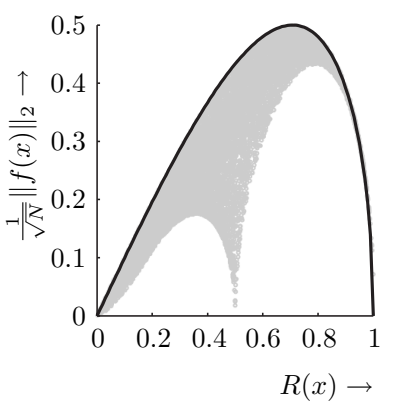

(b) $N=4$

Figure 2: Scatter plot of $\frac{1}{\sqrt{N}}\|f(x)\|_{2}$ for $N=3$ (left panel) and $N=4$ (right panel). The phases $x$ were drawn from a uniform distribution. The solid black line in both panels is the upper bound $R(x) \sqrt{1-R^{2}(x)}$.

Figure 2 illustrates the result of Lemma 4. When $N=4$ (even), the lower bound is attained at every value of $R(x)$, which shows that the given bound is the tightest possible. However, as illustrated in the left panel, when $N=3$, the bound is never attained except on the set $\left\{x \in \mathbb{R}^{N}: R(x) \in\{0,1\}\right\}$. It can be shown however that in the limit of large $N$ the given bound is arbitrarily tight, even for odd $N$, in the sense that for every $c \in[0,1]$,

$$
\min _{\left\{x \in \mathbb{R}^{2 m+1}: R(x)=c\right\}} \frac{1}{\sqrt{2 m+1}}\left|\|f(x)\|_{2}-\sqrt{(2 m+1) c^{2}\left(1-c^{2}\right)}\right|
$$


tends to zero as $m$ tends to infinity.

Lemma 4 has some interesting implications. For instance, it can provide insight into the rate at which solutions of a homogeneous system of Kuramoto oscillators $\left((2)\right.$ with $\omega_{i}=0$ for $\left.1 \leq i \leq N\right)$ on $\mathbb{R}^{N}$ converge to fixed points, . To see this, consider the homogeneous system

$$
\left\{\begin{array}{ccc}
\dot{\theta}(t) & = & k f(\theta(t)) \\
\theta\left(t_{0}\right) & = & \theta_{0}
\end{array}\right.
$$

where $\theta_{0} \in \mathbb{R}^{N}$. We shall compute the time derivative of the magnitude squared of the order parameter, $L(\cdot):=R^{2}(\cdot)$, and show that this derivative is: $(i)$ nonnegative along solutions of $(40) ;(i i)$ bounded from above by a certain function $D(t)$ for every $t$. We proceed as follows [9]. By definition,

$$
\frac{\mathrm{d} L(\theta(t))}{\mathrm{d} t}:=\frac{L(\theta)}{\partial \theta} \dot{\theta}(t)=\frac{L(\theta)}{\partial \theta} k f(\theta(t)) .
$$

Using the identity

$$
\frac{\partial L(\theta)}{\partial \theta}=\frac{2}{N}[f(\theta)]^{T}
$$

it follows that

$$
\frac{\mathrm{d} L(\theta(t))}{\mathrm{d} t}=\frac{2 k}{N}\|f(\theta(t))\|_{2}^{2}
$$

which shows that the time-derivative is positive everywhere, except at the equilibria, where it is zero. It follows that the magnitude of the order parameter is a nondecreasing function of time. Based on the observation that the timederivative of $L$ is positive almost everywhere (the set of equilibria having measure zero), we formulate the following conjecture, $[3,9]$ :

Conjecture 1 For almost all initial conditions $\theta_{0}$, the solution $\theta(t)$ to the homogeneous system (40) has the property that $\lim _{t \rightarrow \infty} R(\theta(t))=1$.

In agreement with Conjecture 1, one can prove that, for the homogeneous system, the global phase-locking manifold $\mathcal{M}:=\left\{\theta \in \mathbb{R}^{N}: \theta_{i}=\theta_{j}\right.$ for all $\left.i, j\right\}$ is (locally) asymptotically stable. However, the existence of other invariant manifolds, not contained in $\mathcal{M}$, implies that $\mathcal{M}$ is not globally asymptotically stable. We conjecture that $\mathcal{M}$ is 'almost globally asymptotically stable', in the sense that its region of attraction is the entire space minus a set of measure zero.

For our next result, we shall need the concept of a dominating function, which is defined as follows:

Definition 3 Let $h, g: \mathbb{R} \mapsto \mathbb{R}$ and let $\mathcal{I} \subset \mathbb{R}$ be some interval. We say that $h$ dominates $g$ on $\mathcal{I}$ if $h(t) \geq g(t)$ for all $t \in \mathcal{I}$. In that case we call $h$ a dominating function for $g$ on $\mathcal{I}$.

Our next result states that $L(\theta(t))$ is dominated by a certain scalar function $D(t)$ that depends only on $\theta_{0}$. In order to prove this result, we need the following two lemmas. 
Lemma 5 Let $\theta(\cdot)$ be a solution of the homogeneous system (40) and suppose $\dot{L}\left(\theta\left(t^{\prime}\right)\right)=2 k L\left(\theta\left(t^{\prime}\right)\right)\left(1-L\left(\theta\left(t^{\prime}\right)\right)\right)$ for some $t^{\prime} \in \mathbb{R}$. Then

$$
\dot{L}(\theta(t))=2 k L(\theta(t))(1-L(\theta(t))) \quad \text { for all } t \geq t^{\prime} .
$$

Proof: Recall that $\dot{L}(\theta(t))=\frac{2 k}{N}\|f(\theta(t))\|_{2}^{2}$. It follows from the proof of Lemma 4 that $\left\|f\left(\theta\left(t^{\prime}\right)\right)\right\|_{2}^{2}=N L\left(\theta\left(t^{\prime}\right)\right)\left(1-L\left(\theta\left(t^{\prime}\right)\right)\right)$ for some $t^{\prime} \in \mathbb{R}$ if and only if one or two of the following conditions hold: (a) $L\left(\theta\left(t^{\prime}\right)\right)=0$; (b) $N$ is even and there exists a permutation $\hat{\theta}\left(t^{\prime}\right)$ of $\theta\left(t^{\prime}\right)$ such that

$$
\begin{aligned}
\cos \left(\hat{\theta}_{i}\left(t^{\prime}\right)-\hat{\theta}_{1}\left(t^{\prime}\right)\right)=1, & i=1,2, \ldots, \frac{N}{2} \\
\cos \left(\hat{\theta}_{i}\left(t^{\prime}\right)-\hat{\theta}_{N}\left(t^{\prime}\right)\right)=1, & i=\frac{N}{2}+1, \ldots, N
\end{aligned}
$$

If $L\left(\theta\left(t^{\prime}\right)\right)=0$, we have that $L(\theta(t))=0$ for all $t \geq t^{\prime}$ and the result follows trivially. Now suppose Conditions (42) and (43) hold. It follows that $\dot{\hat{\theta}}_{i}\left(t^{\prime}\right)=$ $\dot{\hat{\theta}}_{j}\left(t^{\prime}\right)$ for $i, j \leq \frac{N}{2}$ and $i, j>\frac{N}{2}$, and hence

$$
\left.\frac{\mathrm{d}}{\mathrm{d} \tau}\left(\cos \left(\hat{\theta}_{i}(\tau)-\hat{\theta}_{j}(\tau)\right)\right)\right|_{\tau=t^{\prime}}=0, \quad i, j \leq \frac{N}{2} ; i, j>\frac{N}{2}
$$

In other words, if $\hat{\theta}(\cdot)$ satisfies Conditions (42) and (43) for some $t^{\prime}$, it satisfies (42) and (43) for all $t \geq t^{\prime}$. This concludes the proof.

Lemma 6 Let $h, g: \mathbb{R} \mapsto \mathbb{R}$ be such that for every $x_{0} \in \mathbb{R}$, the systems

$$
\left\{\begin{array}{ccc}
\dot{x} & = & h(x) \\
x(0) & = & x_{0}
\end{array}, \quad\left\{\begin{array}{ccc}
\dot{x} & = & g(x) \\
x(0) & = & x_{0}
\end{array}\right.\right.
$$

have unique solutions in $C^{1}[0, \infty)$. Let these solution be denoted $x_{h}\left(t ; x_{0}\right)$ and $x_{g}\left(t ; x_{0}\right)$, respectively. Suppose there exists $a, b \in \mathbb{R}, a \neq b$, such that

$$
h(x)>g(x) \geq 0
$$

for all $x \in(a, b) \subset \mathbb{R}$. Then for every $x_{0} \in(a, b)$, we have that

$$
x_{h}\left(t ; x_{0}\right) \geq x_{g}\left(t ; x_{0}\right)
$$

for all $t \in I$, where $I \subset \mathbb{R}$ is defined as

$$
I:= \begin{cases}{\left[0, \min _{t}\left\{x_{h}\left(t ; x_{0}\right)=b\right\}\right)} & \text { when }\left\{x_{h}\left(t ; x_{0}\right)=b\right\} \neq \emptyset ; \\ {[0, \infty)} & \text { otherwise. }\end{cases}
$$

Proof: Under the hypotheses of the lemma we have that $h\left(x_{0}\right)>g\left(x_{0}\right)$, and it follows that for small enough $t, x_{h}(t)>x_{g}(t)$ (omitting the argument $x_{0}$ for notational convenience). Note also that $x_{h}$ and $x_{g}$ are increasing whenever $x_{h}\left(t ; x_{0}\right)<b$ and $x_{g}\left(t ; x_{0}\right)<b$ respectively. Now suppose there exists $t_{2}>0$ such that $x_{g}\left(t_{2}\right)>x_{h}\left(t_{2}\right)$ and $x_{g}\left(t_{2}\right)<b$. Then by continuity there exists $t_{1}<t_{2}$ such that $a<x_{h}\left(t_{1}\right)=x_{g}\left(t_{1}\right)<b$ and $h\left(x_{h}\left(t_{1}\right)\right) \leq g\left(x_{g}\left(t_{1}\right)\right)$. Define $x^{\prime}:=x_{h}\left(t_{1}\right)=x_{g}\left(t_{1}\right)$. It follows that $g\left(x^{\prime}\right) \geq h\left(x^{\prime}\right)$, which contradicts our starting hypothesis. We conclude that $x_{h}(t) \geq x_{g}(t)$ for all $t \in I$. 
We have the following result:

Corollary 1 Let $\theta(\cdot)$ be a solution to the homogeneous system (40) with initial condition $\theta\left(t_{0}\right)=\theta_{0}$. Then

$$
D(t):=\frac{1}{1-e^{-2 k\left(t-t_{0}\right)}\left(\frac{L\left(\theta_{0}\right)-1}{L\left(\theta_{0}\right)}\right)}
$$

is a dominating function for $L(\theta(t))$ on $\left[t_{0}, \infty\right)$.

Proof: By Lemma 4 we have that $\dot{L}(\theta(t)) \leq 2 k L(\theta(t))(1-L(\theta(t)))$ for all $t$. We claim that, on $\left[t_{0}, \infty\right), L(\theta(t))$ is dominated by the solution $y(t)$ of the ODE

which is given as

$$
\left\{\begin{array}{ccc}
\dot{y} & = & 2 k y(1-y) \\
y\left(t_{0}\right) & = & L\left(\theta_{0}\right)
\end{array}\right.
$$

$$
y(t)=\frac{1}{1-e^{-2 k\left(t-t_{0}\right)}\left(\frac{L\left(\theta_{0}\right)-1}{L\left(\theta_{0}\right)}\right)}, \quad t \geq t_{0} .
$$

To prove this, suppose $\dot{L}(\theta(t))=2 k L(\theta(t))(1-L(\theta(t)))$ for some $t \geq t_{0}$ and let $t^{\prime}$ denote the smallest such $t$ (in case $\dot{L}(\theta(t))<2 k L(\theta(t))(1-L(\theta(t)))$ for all $t \geq t_{0}$, the result follows immediately from Lemma 6$)$. Then by Lemma 5 , we have that $\dot{L}(\theta(t))=2 k L(\theta(t))(1-L(\theta(t)))$ for all $t \geq t^{\prime}$, and it follows that

$$
\left.L(\theta(t))\right|_{L\left(\theta\left(t^{\prime}\right)\right)=a}=\frac{1}{1-e^{-2 k\left(t-t^{\prime}\right)}\left(\frac{a-1}{a}\right)}, \quad t \geq t^{\prime} .
$$

One can easily verify that $\left.L(\theta(t))\right|_{L\left(\theta\left(t^{\prime}\right)\right)=a}$ is nondecreasing as a function of $a$ for all $t \geq t^{\prime}$ (and $a \in[0,1]$ ). Now let $l$ be an upper bound for $L\left(\theta\left(t^{\prime}\right)\right.$ ). It follows that $\frac{1}{1-e^{-2 k\left(t-t^{\prime}\right)}\left(\frac{l-1}{l}\right)}$ is a dominating function for $L(\theta(t))$ on the interval $\left[t^{\prime}, \infty\right)$. To compute an upper bound for $L\left(\theta\left(t^{\prime}\right)\right.$, we proceed as follows. By definition of $t^{\prime}$, we have that $\dot{L}(\theta(t))<2 k L(\theta(t))(1-L(\theta(t)))$ for all $t<t^{\prime}$. It follows from Lemma 6 that

$$
L(\theta(t)) \leq \frac{1}{1-e^{-2 k\left(t-t_{0}\right)}\left(\frac{L\left(\theta_{0}\right)-1}{L\left(\theta_{0}\right)}\right)}, \quad t_{0} \leq t<t^{\prime}
$$

By continuity, we have that

$$
L\left(\theta\left(t^{\prime}\right)\right) \leq \frac{1}{1-e^{-2 k\left(t^{\prime}-t_{0}\right)}\left(\frac{L\left(\theta_{0}\right)-1}{L\left(\theta_{0}\right)}\right)} .
$$

Using the upper bound $\frac{1}{1-e^{-2 k\left(t^{\prime}-t_{0}\right)}\left(\frac{L\left(\theta_{0}\right)-1}{L\left(\theta_{0}\right)}\right)}$ for $L\left(\theta\left(t^{\prime}\right)\right)$, it follows from (49) that

$$
L(\theta(t)) \leq \frac{1}{1-e^{-2 k\left(t-t_{0}\right)}\left(\frac{L\left(\theta_{0}\right)-1}{L\left(\theta_{0}\right)}\right)}, \quad t \geq t^{\prime}
$$


Combining (50) and (51), we arrive at the desired result. This concludes the proof.

Figure 3 shows the graph of $L(\theta(t))$ and that of the dominating function $D(t)$-Eqn. (46) for a particular realization of the initial condition $\theta_{0}$. In this example, $N=100$ and $k=2$. We observe that, in agreement with Conjecture 1 , the solution converges to a globally phase-locked state, that is $L((\theta(t)) \rightarrow 1$. Note that convergence can be very slow depending on the choice of initial condition. Indeed, for any $T \in \mathbb{R}$ and any $\epsilon>0$, we can find $\delta>0$ such that if $L\left(\theta_{0}\right)<\delta$ then $L(\theta(t))<\epsilon$ for all $t \leq t_{0}+T$. The upshot of this is that if the initial condition $\theta_{0}$ is selected by drawing from a uniform distribution and the number of oscillators is large, then $L\left(\theta_{0}\right)$ is likely to be small, and as a consequence convergence to the stable equilibrium is likely to be slow. In the limit case when $N$ tends to infinity, we have that $L\left(\theta_{0}\right)$ tends to zero with probability 1 and the time required for $L(\theta(t))$ to exceed some given finite threshold diverges to infinity.

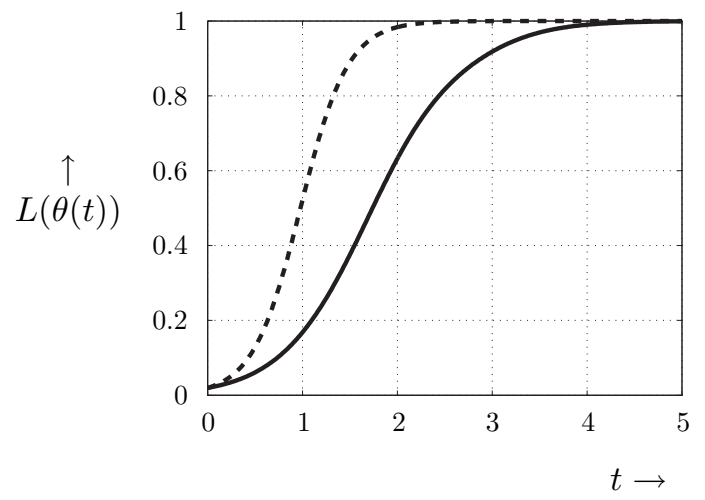

Figure 3: Numerical simulation of the homogeneous system (40) with $N=100$ oscillators and coupling coefficient $k=2$ : time evolution of $L(\theta(t)):=R^{2}(\theta(t))$ (solid line) and the dominating function $D(t)$-Eqn. (46) (dashed line).

Let $\sigma_{\omega}:=\sqrt{\frac{1}{N} \sum_{j=1}^{N}\left(\omega_{j}-\langle\omega\rangle\right)^{2}}$ denote the (sample) standard deviation associated with the vector of intrinsic frequencies $\omega$. Using Lemma 4 we derive another lower bound on the critical coupling, as follows:

Corollary 2 The critical coupling $k_{c}$ satisfies

$$
k_{c} \geq 2 \sigma_{\omega}
$$

Proof: Let $x^{*} \in V \mathbb{R}^{N}$ be a fixed point of the system (17). Then by definition $k\left\|f\left(x^{*}\right)\right\|_{2}=\|V \omega\|_{2}=\sqrt{N} \sigma_{\omega}$ and by Lemma 4 we have that

$$
\left\|f\left(x^{*}\right)\right\|_{2} \leq \sqrt{N} \sqrt{R^{2}\left(x^{*}\right)\left(1-R^{2}\left(x^{*}\right)\right)} .
$$




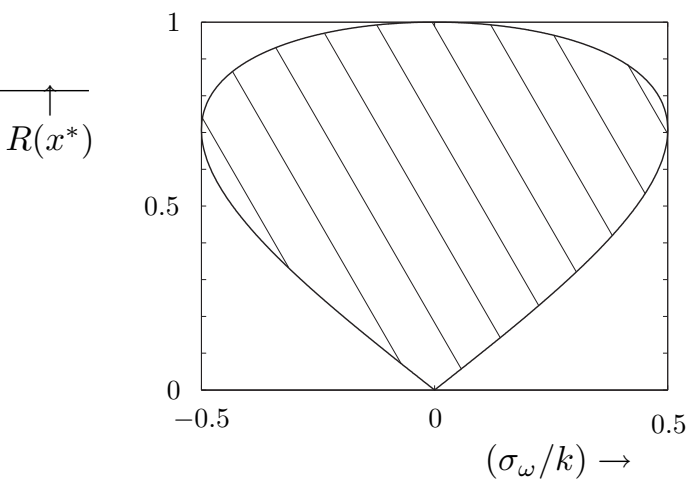

Figure 4: Graph associated with inequality (56). For a given value of the ratio $\left(\sigma_{\omega} / k\right)$, the magnitude of the order parameter $R(\cdot)$, evaluated at a fixed point $x^{*}$, must lie within the striped region.

It is not hard to see that the right hand side of (53) is upper bounded by $\frac{1}{2} \sqrt{N}$. It follows that

$$
k \geq \frac{\sqrt{N} \sigma_{\omega}}{\sqrt{N} \frac{1}{2}}=2 \sigma_{\omega}
$$

This completes the proof.

Note that Corollary 2 is in agreement with the intuition that greater variation in intrinsic frequencies requires stronger coupling to achieve global phase-locking.

Using Lemma 4 we can compute bounds on the value of the order parameter evaluated at the fixed points of the system, should they exist. Indeed, suppose $k>k_{\mathrm{c}}$, then for any fixed point $x^{*} \in V \mathbb{R}^{N}$ we have that

$$
\sqrt{R^{2}\left(x^{*}\right)\left(1-R^{2}\left(x^{*}\right)\right)} \geq \frac{\sigma_{\omega}}{k} .
$$

Solving for $R\left(x^{*}\right)$ gives

$$
\frac{1}{2}-\frac{1}{2} \sqrt{1-4\left(\frac{\sigma_{\omega}}{k}\right)^{2}} \leq R^{2}\left(x^{*}\right) \leq \frac{1}{2}+\frac{1}{2} \sqrt{1-4\left(\frac{\sigma_{\omega}}{k}\right)^{2}}
$$

The graph associated with inequality (56) is shown in Figure 4.

\section{$5 \quad$ Necessary and Sufficient conditions}

In the last section, we derived lower bounds for the critical coupling of the system (17), which provide necessary conditions for the existence of fixed points. We next derive conditions that are both necessary and sufficient for fixed points to exist, and we shall use these results to describe an algorithm for computing the critical coupling in Section 6. 
Throughout this section, the function $f: \mathbb{R}^{N} \mapsto \mathbb{R} R^{N}$ is given by (4) and the set $\mathcal{F}(k, \Omega)$ is defined as

$$
\mathcal{F}(k, \Omega):=\left\{x \in V \mathbb{R}^{N}: k f(x)=-\Omega\right\}, \quad k \geq 0, \quad \Omega \in V \mathbb{R}^{N}
$$

On $\mathcal{F}(k, \Omega)$ we introduce a notion of equivalence, as follows.

Definition 4 (Equivalence on $\mathcal{F}(k, \Omega)$ ) Given $\Omega \in V \mathbb{R}^{N}$ and $k \geq 0$, let $x, x^{\prime} \in F(k, \Omega)$. We say that $x$ and $x^{\prime}$ are equivalent $\left(x \simeq x^{\prime}\right)$ if $R(x)=R\left(x^{\prime}\right)$.

To motivate this definition consider the following fact. Let $k, \Omega$ be given, and let $s \in \mathbb{Z}^{N}$ be such that $\sum_{j=1}^{N} s_{j}=0$. If $x$ is a fixed point of the system (17), then $x^{\prime}:=x+2 s \pi$ is also a fixed point of the system (17) and, in addition, $R\left(x^{\prime}\right)=R(x)$.

The following theorem provides a necessary and sufficient condition for the system (17) to have a fixed point, given a particular coupling strength $k$, and a particular realization of intrinsic frequencies, $\Omega$.

Theorem 2 Let $k>0$ and $\Omega \in V \mathbb{R}^{N}$. Then $\mathcal{F}(k, \Omega) \neq \emptyset$ iff there exists $\beta \in\left[\frac{1}{k}\|\Omega\|_{\infty}, 1\right] \subset \mathbb{R}$ and $a \in\{-1,1\}^{N}$ such that

$$
\beta=\frac{1}{N} \sum_{j=1}^{N} a_{j} \sqrt{1-\left(\frac{\Omega_{j}}{k \beta}\right)^{2}} .
$$

Moreover, suppose $\left(a^{1}, \beta^{1}\right)$ and $\left(a^{2}, \beta^{2}\right)$ both satisfy (57) and let $x^{1}, x^{2} \in F(k, \Omega)$ be such that

$$
\left\{\begin{array}{rl}
k \beta^{i} \sin \left(\psi\left(x^{i}\right)-x_{j}^{i}\right) & =-\Omega_{j} \\
a_{j} \cos \left(\psi\left(x^{i}\right)-x_{j}^{i}\right) & \geq 0
\end{array}, \quad i \in\{1,2\}, \quad j=1,2, \ldots N\right.
$$

Then $x^{1} \simeq x^{2}$ iff $\beta^{1}=\beta^{2}$ and $\sum_{j=1}^{N}\left(a_{i}-a_{j}\right) \sqrt{1-\left(\frac{\Omega_{j}}{k \beta^{1}}\right)^{2}}=0$.

Proof: $\quad$ Suppose $\Omega \neq 0$ (the case $\Omega=0$ is easy). Let $x^{*} \in V \mathbb{R}^{N}$ be a fixed point of (17). By definition, $k f\left(x^{*}\right)=-\Omega$, and since $\Omega \neq 0$, we have that $f\left(x^{*}\right) \neq 0$, and consequently $R\left(x^{*}\right) \neq 0$. It follows that

$$
\sin \left(\psi\left(x^{*}\right)-x_{i}^{*}\right)=-\frac{\Omega_{i}}{k R\left(x^{*}\right)}, \quad i=1,2, \ldots, N .
$$

Let $\beta:=R\left(x^{*}\right)$. By (59) we have that $\beta \geq \frac{1}{k}\|\Omega\|_{\infty}$. Recall that for all $x \in$ $\mathbb{R}^{N} \backslash \mathcal{R}_{0}, R(x)$ can be written as

$$
R(x)=\frac{1}{N} \sum_{j=1}^{N} \cos \left(\psi(x)-x_{j}\right)
$$

and let $a_{i}$ be given as

$$
a_{i}:= \begin{cases}-1 & \text { if } \cos \left(\psi\left(x^{*}\right)-x_{i}^{*}\right) \leq 0 \\ +1 & \text { otherwise }\end{cases}
$$


Combining (59), (60), and (61), we arrive at

$$
\beta=\frac{1}{N} \sum_{j=1}^{N} a_{j} \sqrt{1-\left(\frac{\Omega_{j}}{k \beta}\right)^{2}} .
$$

This proves necessity. To prove sufficiency, let $a \in\{-1,1\}^{N}$ be given, and suppose $\beta \geq \frac{1}{k}\|\Omega\|_{\infty}>0$ (again, the case $\Omega=0$ is easy). Then for every $c \in \mathbb{R}$, the system

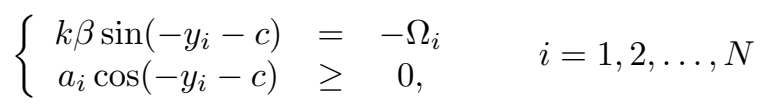

has a unique solution $y^{*} \in[-\pi, \pi)^{N}$. We pick $c$ such that $\sum_{j=1}^{N} y_{j}^{*}=0$. Since $\sum_{j=1}^{N} \sin \left(y_{j}^{*}+c\right)=0$, it follows that

$$
R\left(y^{*}\right)=R\left(y^{*}+c \mathbf{1}\right)=\left|\sum_{j=1}^{N} \cos \left(y_{j}^{*}+c\right)\right|
$$

From (63), we have that

$$
\cos \left(y_{i}^{*}+c\right)=a_{i} \sqrt{1-\left(\frac{\Omega_{i}}{k \beta}\right)^{2}} \quad i=1, \ldots, N .
$$

Combining (64) and (65), we arrive at

$$
R\left(y^{*}\right)=\left|\frac{1}{N} \sum_{j=1}^{N} a_{j} \sqrt{1-\left(\frac{\Omega_{j}}{k \beta}\right)^{2}}\right|
$$

The second part of the theorem follows easily after noting that if $x^{i}$ satisfies (58) then $R\left(x^{i}\right)=\beta^{i}, i=1,2$.

Theorem 2 gives us a necessary and sufficient condition for the equation $k f(x)=-\Omega$ to have at least one solution for a given value of $k$. It is not clear, however, that there exists a $k$ for which this condition is satisfied. The following Corollary provides an easy sufficient condition.

Corollary 3 Let $k>0$ and $\Omega \in V \mathbb{R}^{N}$. Suppose

$$
\frac{1}{k}\|\Omega\|_{\infty} \leq \frac{1}{N} \sum_{j=1}^{N} a_{j} \sqrt{1-\left(\frac{\Omega_{j}}{\|\Omega\|_{\infty}}\right)^{2}} .
$$

for some $a \in\{-1,1\}^{N}$. Then $\mathcal{F}(k, \Omega) \neq \emptyset$.

Proof: Suppose $\Omega \neq 0$ (again, the case $\Omega=0$ is easy). Let $a \in\{-1,1\}^{N}$. Define $m:\left[\frac{1}{k}\|\Omega\|_{\infty}, 1\right] \mapsto \mathbb{R}, m(\beta):=\beta$ and $n:\left[\frac{1}{k}\|\Omega\|_{\infty}, 1\right] \times\{-1,1\}^{N} \mapsto \mathbb{R}$,

$$
n(\beta, a):=\frac{1}{N} \sum_{j=1}^{N} a_{j} \sqrt{1-\left(\frac{\Omega_{j}}{k \beta}\right)^{2}} .
$$


Since $\Omega \neq 0$ we have that $m(1)>n(1, a)$. Now suppose condition $(67)$ is satisfied. Then we have that $m\left(\frac{1}{k}\|\Omega\|_{\infty}\right) \leq n\left(\frac{1}{k}\|\Omega\|_{\infty}, a\right)$, and by the Intermediate Value Theorem there must exist $\beta^{*} \in\left[\frac{1}{k}\|\Omega\|_{\infty}, 1\right]$ such that $m\left(\beta^{*}\right)=n\left(\beta^{*}, a\right)$. It follows from Theorem 2 that the system (17) has a fixed point.

Corollary 4 Let $\Omega \in V \mathbb{R}^{N}$. Then: (i) the critical coupling $k_{c}$ is finite; (ii) for large enough coupling, the system (17) has at least $2^{N-1}$ fixed points.

Proof: Note that the right hand side of (67) does not depend on $k$. Hence, it follows that, provided

$$
\frac{1}{N} \sum_{j=1}^{N} a_{j} \sqrt{1-\left(\frac{\Omega_{j}}{\|\Omega\|_{\infty}}\right)^{2}}>0
$$

Condition (67) is always satisfied for large enough $k$. Furthermore, it follows easily that if (69) is not satisfied for given $a$, then it is satisfied for $a^{\prime}:=-a$. This implies $(i)$ that the critical coupling $k_{\mathrm{c}}$ is always finite, and $(i i)$ that the set $A^{+}:=\left\{a \in\{-1,1\}^{N}\right.$ : Eqn. (69) is satisfied $\}$ contains precisely $2^{N-1}$ elements (counting multiplicity), each of which defines a unique (up to equivalence in the sense of Definition 4) fixed point. This concludes the proof.

Corollary 5 Let $k>0$ and $\Omega \in V \mathbb{R}^{N}$. Then $\mathcal{F}(k, \Omega) \neq \emptyset$ if and only if there exist $\beta \in\left[\frac{1}{k}\|\Omega\|_{\infty}, 1\right]$ such that

$$
\beta=\frac{1}{N} \sum_{j=1}^{N} \sqrt{1-\left(\frac{\Omega_{j}}{k \beta}\right)^{2}} .
$$

Proof: The proof of Corollary 3 suggests that if the fixed point equation (57) does not have a solution, then necessarily

$$
\beta>\frac{1}{N} \sum_{j=1}^{N} a_{j} \sqrt{1-\left(\frac{\Omega_{j}}{k \beta}\right)^{2}}
$$

for all $\beta \in\left[\frac{1}{k}\|\Omega\|_{\infty}, 1\right]$ and for all $a \in\{-1,1\}^{N}$. Since we have that

$$
\frac{1}{N} \sum_{j=1}^{N} \sqrt{1-\left(\frac{\Omega_{j}}{k \beta}\right)^{2}} \geq \frac{1}{N} \sum_{j=1}^{N} a_{j} \sqrt{1-\left(\frac{\Omega_{j}}{k \beta}\right)^{2}}
$$

for all $a \in\{-1,1\}^{N}$, it follows that the given condition is necessary and sufficient for the system (17) to have at least one fixed point. This concludes the proof.

The next and final corollary gives us an upper bound on the critical coupling.

Corollary 6 The critical coupling, $k_{c}$, satisfies:

$$
k_{c} \leq \frac{\|\Omega\|_{\infty}}{\frac{1}{N} \sum_{j=1}^{N} \sqrt{1-\left(\frac{\Omega_{j}}{\|\Omega\|_{\infty}}\right)^{2}}} .
$$

Proof: Follows directly from Corollary 3. 


\section{An algorithm for computing $k_{\mathrm{c}}$}

In this section we present a bisection algorithm that will allow us to numerically evaluate the critical coupling with arbitrary precision. Throughout, we shall assume that $\Omega \neq 0$. Define $\mathcal{I}:=\left(\|\Omega\|_{\infty}, \infty\right)$, and let $p_{i}: \mathcal{I} \mapsto(0,1]$, and $P: \mathcal{I} \mapsto(0,1]$ be given as

$$
p_{i}(u):=\sqrt{1-\left(\frac{\Omega_{i}}{u}\right)^{2}} \quad ; \quad P(u):=\frac{1}{N} \sum_{j=1}^{N} p_{j}(u)
$$

Also, define $h(u ; k): \mathcal{I} \times \mathbb{R}_{+} \mapsto \mathbb{R}_{+}$,

$$
h(u ; k):=\frac{1}{k} u .
$$

From Corollary 5 it follows that the critical coupling is the smallest $k$ for which the equation $P(u)=h(u ; k)$ has at least one solution on $\mathcal{I}$. We have the following result.

Theorem 3 For all $\Omega \in V \mathbb{R}^{N}, \Omega \neq 0$, the equation

$$
2 \frac{1}{N} \sum_{j=1}^{N} \sqrt{1-\left(\frac{\Omega_{j}}{u}\right)^{2}}=\frac{1}{N} \sum_{j=1}^{N} \frac{1}{\sqrt{1-\left(\frac{\Omega_{j}}{u}\right)^{2}}} .
$$

has a unique solution $u^{*} \in \mathcal{I}$, and we have that

$$
k_{c}=\frac{u^{*}}{\frac{1}{N} \sum_{j=1}^{N} \sqrt{1-\left(\frac{\Omega_{j}}{u^{*}}\right)^{2}}} .
$$

Proof: Observe that, by strict concavity of $P$ and linearity of $h(\cdot ; k)$, the equation $P(u)=h(u ; k)$ can have at most two solutions on $\mathcal{I}$ for any $k>0$. We shall now show that, when $k=k_{\mathrm{c}}$, it can have no more than one solution. Since, by definition of critical coupling, $P(u)=h\left(u ; k_{\mathrm{c}}\right)$ must have at least one solution, we shall conclude that it has precisely one solution. Let $k=k_{\mathrm{c}}$ and suppose there exist $u^{1}, u^{2} \in \mathcal{I}, u^{1} \neq u^{2}$, such that $P\left(u^{1}\right)=h\left(u^{1} ; k_{\mathrm{c}}\right)$ and $P\left(u^{2}\right)=h\left(u^{2} ; k_{\mathrm{c}}\right)$. By strict concavity of $P$ we have that $P\left(\frac{1}{2}\left(u^{1}+u^{2}\right)\right)>\frac{1}{2}\left(P\left(u^{1}\right)+P\left(u^{2}\right)\right)$. Define $u^{\prime}:=\frac{1}{2}\left(u^{1}+u^{2}\right)$ and note that $u^{\prime} \in \mathcal{I}$. We have that $P\left(u^{\prime}\right)>h\left(u^{\prime} ; k_{\mathrm{c}}\right)$. This implies that there exists $k^{\prime}<k_{\mathrm{c}}$ such that $P\left(u^{\prime}\right)=h\left(u^{\prime} ; k^{\prime}\right)$. But by definition $k_{\mathrm{c}}$ is the smallest $k$ for which $P(u)=h(u ; k)$ has a solution. We arrive at a contradiction and conclude that $u^{1}=u^{2}$; or in other words, that the equation $P(u)=h\left(u ; k_{\mathrm{c}}\right)$ has exactly one solution on $\mathcal{I}$. Denoting this solution by $u^{*}$, it is not hard to see that, at $u=u^{*}$, the derivative of $P$ with respect to $u$ and the derivative of $h$ with respect to $u$ (both of which are defined on the entire interval $\mathcal{I}$ ) must coincide. For suppose $\frac{\partial h}{\partial u}\left(u^{*}\right)<\frac{\partial P}{\partial u}\left(u^{*}\right)$, then by continuity there exists $\delta>0$ such that $h\left(u ; k_{\mathrm{c}}\right)<P(u)$ for all $u$ such that $u-u^{*}<\delta$. Let 
$u^{\prime}$ be one such $u$. It follows that there exists $k^{\prime}<k_{\mathrm{c}}$ such that $P\left(u^{\prime}\right)=h\left(u^{\prime} ; k^{\prime}\right)$. This leads to a contradiction and we conclude that $h\left(u ; k_{\mathrm{c}}\right) \geq P(u)$. By analogy we have that $h\left(u ; k_{\mathrm{c}}\right) \leq P(u)$. We conclude that $\frac{\partial h}{\partial u}\left(u^{*}\right)=\frac{\partial P}{\partial u}\left(u^{*}\right)$. That is,

$$
\frac{1}{k_{\mathrm{c}}}=\frac{1}{u^{*}} \frac{1}{N} \sum_{j=1}^{N} \frac{\left(\frac{\Omega_{j}}{u^{*}}\right)^{2}}{\sqrt{1-\left(\frac{\Omega_{j}}{u^{*}}\right)^{2}}} .
$$

Or equivalently,

$$
\begin{aligned}
\frac{u^{*}}{k_{\mathrm{c}}} & =\frac{1}{N} \sum_{j=1}^{N} \frac{\left(\frac{\Omega_{j}}{u^{*}}\right)^{2}}{\sqrt{1-\left(\frac{\Omega_{j}}{u^{*}}\right)^{2}}} \\
& =-\frac{1}{N} \sum_{j=1}^{N} \sqrt{1-\left(\frac{\Omega_{j}}{u^{*}}\right)^{2}}+\frac{1}{N} \sum_{j=1}^{N} \frac{1}{\sqrt{1-\left(\frac{\Omega_{j}}{u^{*}}\right)^{2}}}
\end{aligned}
$$

Now recall that by definition of $u^{*}$, we have that

$$
\frac{u^{*}}{k_{\mathrm{c}}}=\frac{1}{N} \sum_{j=1}^{N} \sqrt{1-\left(\frac{\Omega_{j}}{u^{*}}\right)^{2}} .
$$

Equating the right hand side of Eqn. (76) with the right hand side of Eqn. (77) gives

$$
2 \frac{1}{N} \sum_{j=1}^{N} \sqrt{1-\left(\frac{\Omega_{j}}{u^{*}}\right)^{2}}=\frac{1}{N} \sum_{j=1}^{N} \frac{1}{\sqrt{1-\left(\frac{\Omega_{j}}{u^{*}}\right)^{2}}} .
$$

This shows that $u^{*}$ is a solution to $(73)$. What remains to be shown is that $u^{*}$ is the only solution. Define $v, w: \mathcal{I} \mapsto \mathbb{R}$,

$$
v(u):=2 \frac{1}{N} \sum_{j=1}^{N} \sqrt{1-\left(\frac{\Omega_{j}}{u}\right)^{2}}, \quad w(u):=\frac{1}{N} \sum_{j=1}^{N} \frac{1}{\sqrt{1-\left(\frac{\Omega_{j}}{u}\right)^{2}}},
$$

and note that, on their respective domains, $v$ is strictly monotonically decreasing while $w$ is strictly monotonically increasing. In addition, note that there exist $a, b \in \mathcal{I}$ such that $v(a)>w(a)$ and $v(b)<w(b)$. Hence, by continuity, there must exist a point $u^{\prime} \in(a, b) \subset \mathcal{I}$ such that $v\left(u^{\prime}\right)=w\left(u^{\prime}\right)$. Monotonicity of $v$ and $w$ implies that this point is unique. It follows that $u^{*}$ is the unique solution of $(73)$ on $\mathcal{I}$. And by (77) we have that

$$
k_{\mathrm{c}}=\frac{u^{*}}{\frac{1}{N} \sum_{j=1}^{N} \sqrt{1-\left(\frac{\Omega_{j}}{u^{*}}\right)^{2}}}
$$

This concludes the proof. 
Based on the result of Theorem 3, we define the map $K: V \mathbb{R}^{N} \backslash\{0\} \mapsto \mathbb{R}_{+}$,

$$
K(\Omega)=\frac{u^{*}}{\frac{1}{N} \sum_{j=1}^{N} \sqrt{1-\left(\frac{\Omega_{j}}{u^{*}}\right)^{2}}},
$$

where, as before, $u^{*}$ denotes the unique solution of $(73)$ on $\mathcal{I}$, given $\Omega$. Note that, given any realization of $\omega$ such that $V \omega \neq 0$, we have that $k_{\mathrm{c}}=K(V \omega)$. We have the following corollary.

\section{Corollary 7}

1. For all $\Omega \in V \mathbb{R}^{N}, \Omega \neq 0$, we have that $\|\Omega\|_{\infty} \leq K(\Omega) \leq 2\|\Omega\|_{\infty}$;

2. there exists $\Omega \in V \mathbb{R}^{N}$ such that $K(\Omega)=2\|\Omega\|_{\infty}$ if and only if $N$ is even;

3. for every $\epsilon>0$ there exist an positive integer $N$ and $\Omega \in V \mathbb{R}^{N}$ such that $\left|K(\Omega)-\|\Omega\|_{\infty}\right|<\epsilon$.

Proof: (Part 1). We show that for all $\Omega \neq 0$, the solution $u^{*}$ of equation (73) satisfies $u^{*} \leq \sqrt{2}\|\Omega\|_{\infty}$. The result then follows easily. Let $u^{\prime}:=\sqrt{2}\|\Omega\|_{\infty}$. Then we have that

$$
2 \frac{1}{N} \sum_{j=1}^{N} \sqrt{1-\left(\frac{\Omega_{j}}{u}\right)^{2}}>\frac{1}{N} \sum_{j=1}^{N} \frac{1}{\sqrt{1-\left(\frac{\Omega_{j}}{u}\right)^{2}}} \text { for all } u>u^{\prime}
$$

It follows that

$$
K(\Omega) \leq \frac{u^{\prime}}{\frac{1}{N} \sum_{j=1}^{N} \sqrt{1-\left(\frac{\Omega_{j}}{u^{\prime}}\right)^{2}}} \leq \frac{\sqrt{2}\|\Omega\|_{\infty}}{\frac{1}{2} \sqrt{2}}=2\|\Omega\|_{\infty}
$$

for all $\Omega \in V \mathbb{R}^{N}$. The lower bound $K(\Omega) \geq\|\Omega\|_{\infty}$ was obtained earlier in Section 4 .

(Part 2.) From the above it follows that $K(\Omega)=2\|\Omega\|_{\infty}$ if and only if

$$
2 \frac{1}{N} \sum_{j=1}^{N} \sqrt{1-\left(\frac{\Omega_{j}}{u^{\prime}}\right)^{2}}=\frac{1}{N} \sum_{j=1}^{N} \frac{1}{\sqrt{1-\left(\frac{\Omega_{j}}{u^{\prime}}\right)^{2}}}
$$

or equivalently, $\Omega_{i}^{2}=\Omega_{j}^{2}$ for all $(i, j)$. It is easy to see that this latter condition is never satisfied when $N$ is odd (keeping in mind that $\sum_{j} \Omega_{j}=0$ ). Now suppose $N$ is even and pick any $c \neq 0$. Define

$$
\Omega_{i}:=\left\{\begin{array}{ll}
c & i=1,2, \ldots \frac{N}{2} \\
-c & i=\frac{N}{2}+1, \ldots, N
\end{array} .\right.
$$


Then we have that $\Omega \in V \mathbb{R}^{N}$. Moreover, $\Omega_{i}^{2}=\Omega_{j}^{2}=c^{2}$ for all $(i, j)$. It follows that $K(\Omega)=2\|\Omega\|_{\infty}$.

(Part 3.) Let $\epsilon>0$ be given and suppose $N$ is odd. Pick $c \neq 0$ and define

$$
\Omega_{i}:= \begin{cases}0 & i=1,2, \ldots, N-1 \\ c & i=N .\end{cases}
$$

Then (73) evaluates to

$$
2(N-1)+2 \sqrt{1-\left(\frac{c}{u}\right)^{2}}=(N-1)+\frac{1}{\sqrt{1-\left(\frac{c}{u}\right)^{2}}} .
$$

and it is not hard to see that as $N$ tends to infinity, the solution $u^{*}$ of (81) tends to $c$. Indeed, for $N \geq 2$ we have

$$
\left(\frac{c}{u^{*}}\right)^{2}=\frac{1}{2}(N-1)\left(-\frac{1}{4}(N-1)+\frac{1}{4} \sqrt{(N-1)^{2}+8}\right) .
$$

Let $\epsilon_{1}>0$, and pick $N$ such that $\frac{1}{N}<\epsilon_{1}$ and $u^{*}<\left(1+\epsilon_{1}\right) c$. It follows that

$$
\frac{1}{N} \sum_{j=1}^{N} \sqrt{1-\left(\frac{\Omega_{j}}{u^{*}}\right)^{2}}<1-\epsilon_{1}
$$

and hence

$$
K(\Omega):=\frac{u^{*}}{\frac{1}{N} \sum_{j=1}^{N} \sqrt{1-\left(\frac{\Omega_{j}}{u^{*}}\right)^{2}}}<c\left(\frac{1+\epsilon_{1}}{1-\epsilon_{1}}\right)=c+2\left(\frac{\epsilon_{1}}{1-\epsilon_{1}}\right) c .
$$

Now let $\epsilon_{1}$ be given as $\epsilon_{1}:=\frac{\epsilon}{2 c+\epsilon}$ and choose $N$ accordingly. It follows that $K(\Omega)<\|\Omega\|_{\infty}+\epsilon$. This concludes the proof.

We are now ready to present our algorithm, which, given $\Omega$, will compute $u^{*}$ with user-defined precision $\epsilon>0$ in a finite number of iterations, $n=\left\lceil\log _{2}\left(\frac{\|\Omega\|_{\infty}}{\epsilon}\right)\right\rceil+1$.

\section{Algorithm 1}

1. $a:=\|\Omega\|_{\infty}, b:=\sqrt{2}\|\Omega\|_{\infty}$

2. While $(b-a)>\epsilon$,

3. $u:=\frac{1}{2}(b-a)$.

4. If $\left[\sum_{j} \sqrt{\left(1-\frac{\Omega_{j}}{u}\right)^{2}}>\frac{1}{2} \sum_{j} \frac{1}{\sqrt{\left(1-\frac{\Omega_{j}}{u}\right)^{2}}}\right]$ then $a:=u$, else $b:=u$.

5. End.

Once we have an estimate $\hat{u}$ of $u^{*}$, we can use (74), replacing $u^{*}$ with $\hat{u}$, to estimate $k_{\mathrm{c}}$. 


\section{Numerical Example}

We illustrate the results presented in this paper by means of a numerical example. We consider two systems with $N=20$ and $N=200$ oscillators respectively, with frequencies $\left\{\Omega_{i}^{N}\right\}$ as depicted in Figure 5 . The frequencies in this example

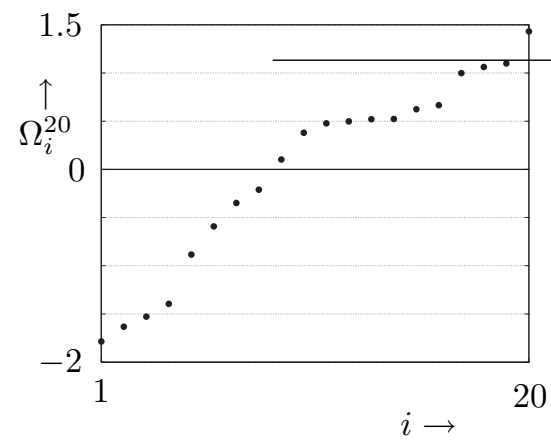

(a) $N=20$

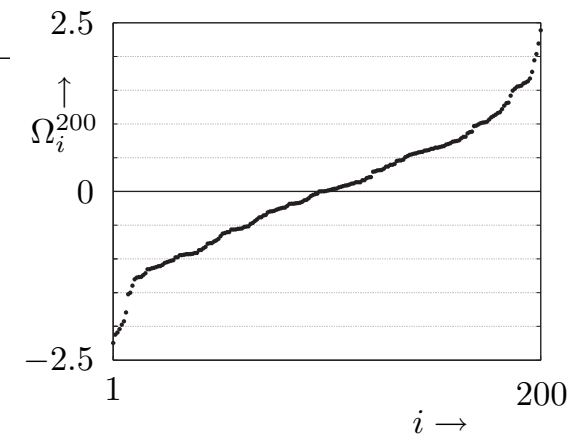

(b) $N=200$

Figure 5: The vector of frequencies $\Omega_{i}^{N}:=\omega_{i}^{N}-\left\langle\omega^{N}\right\rangle, N \in\{20,200\}$, used in this example. The natural frequencies $\omega_{i}^{N}$ were sampled from a normal distribution with zero mean and unit variance and relabelled in such a way that $\omega_{1}^{N} \leq \omega_{2}^{N} \leq \ldots \leq \omega_{N}^{N}$.

were sampled from a normal distribution with zero mean and unit variance and relabelled such that $\omega_{1}^{N} \leq \omega_{2}^{N} \ldots \leq \omega_{N}^{N}$ (note that this can be done without loss of generality). For this particular realization of $\omega^{20}\left(\omega^{200}\right)$, we have that $\left\|\Omega^{20}\right\|_{\infty}=1.7858\left(\left\|\Omega^{200}\right\|_{\infty}=2.3893\right)$ and

$$
\frac{1}{N} \sum_{j=1}^{N} \sqrt{1-\left(\frac{\Omega_{j}}{\|\Omega\|_{\infty}}\right)^{2}}=0.8015 \text { (0.9139). }
$$

It follows from Corollary 6 that $k_{\mathrm{c}} \leq 2.2281(2.6145)$ and by (32), we have that $k_{\mathrm{c}} \geq\|\Omega\|_{\infty}=1.7858$ (2.3893). Figure 7 shows the time evolution of the magnitude squared of the order parameter, $R^{2}(t)$ (previously denoted as $L(t)$ ), for two different initial conditions and two values of the coupling coefficient, $k=2.3$ and $k=2.65(k=2.1$ and $k=2.3)$. We observe that when $k$ is slightly greater than the known lower bound on $k_{\mathrm{c}}$, the value of $R^{2}(t)$ converges to a constant and inspection shows that the solution $x(t)$ of the system (17) tends to a fixed point. On the other hand, when the coupling coefficient is slightly below the known upper bound on the critical coupling, the trajectories $x(t)$ appear not to converge. Note that in this case we do not know whether the system (17) has a fixed point or not, as the condition stated in Corollary 6 is only sufficient while at the same time the respective coupling strengths exceed their known lower bounds (1.7858 and 2.3893 respectively). To gain more insight into this situation let us consider the case $N=20$ in some more detail. We fix the 


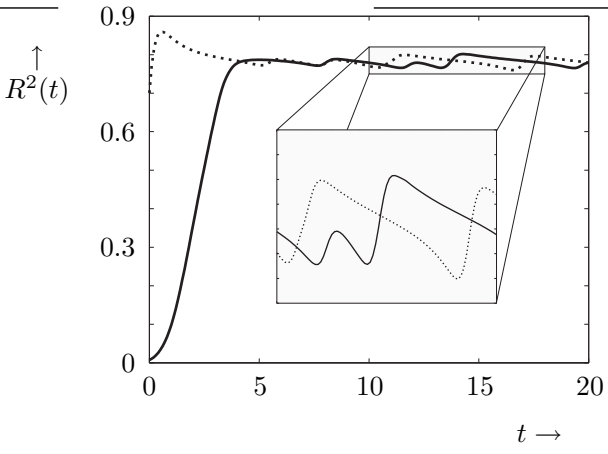

(a) $k=2.4$

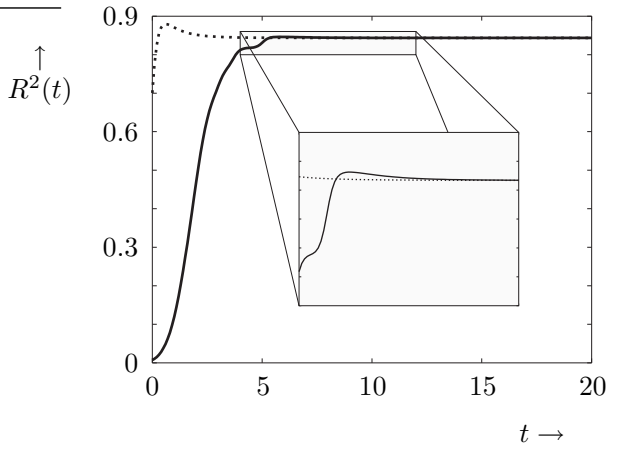

(b) $k=2.65$

Figure 6: Case $N=200$ : time evolution of the magnitude squared of the order parameter, $R^{2}(t)$, for two different initial condition (indicated by a dashed and solid line respectively), and two values of $k$. In the left panel, the value of $k$ (2.4) is (well) below the known upper bound on $k_{\mathrm{c}}$ (2.6145)and the system does not converge to a fixed point; in the right panel the value of $k(2.65)$ is slightly above the known upper bound on $k_{\mathrm{c}}$ and the system converges to a fixed point, as expected.

coupling coefficient at $k=2.1$, and numerically evaluate the function $P^{20}(k, \cdot)$,

$$
P^{20}(k \beta)=\frac{1}{20} \sum_{j=1}^{20} \sqrt{1-\left(\frac{\Omega_{j}^{20}}{k \beta}\right)^{2}},
$$

for several values of $\beta$ in the interval $\left[\frac{1}{k}\left\|\Omega^{20}\right\|_{\infty}, 1\right]$. We repeat the same computation for $k=2.3$. The result is shown in Figure 8 . We observe that the equation $P^{20}(k \beta)=\beta$ does not have a solution on the interval $\left[\frac{1}{k}\left\|\Omega^{20}\right\|_{\infty}, 1\right]$ when $k=2.1$, but does have a solution when $k=2.3$.

We use Algorithm 1 to compute the 'exact' value of the critical coupling to the fifth significant digit. We find that $k_{\mathrm{c}}=2.2198$ for the case $N=20$ and $k_{\mathrm{c}}=2.6144$ for the case $N=200$. Note that in both cases, but particularly the latter, the upper bounds (2.2281 and 2.6145 respectively) provide good estimates of the true values of the critical coupling.

\section{Conclusion}

We derived necessary and sufficient conditions for the existence of fixed points in a finite system of coupled oscillators. In particular, we derived an easy sufficient condition in terms of the individual oscillator frequencies (Corollary 3), which we used to compute an upper bound on the critical coupling (Corollary 6). We showed that when no prior knowledge of the distribution of frequencies is available, we can still bound the critical coupling in terms of the infinity norm 


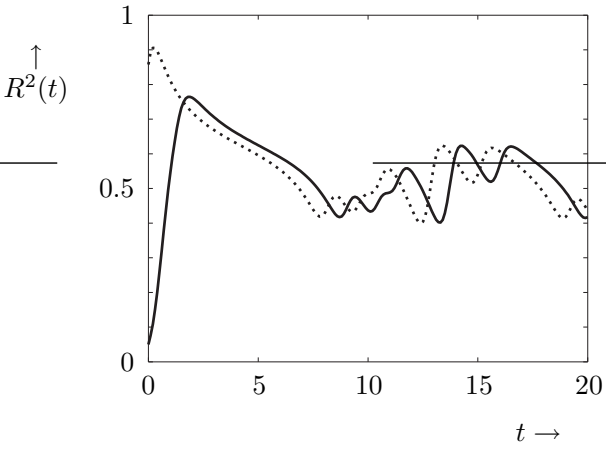

(a) $k=2.1$

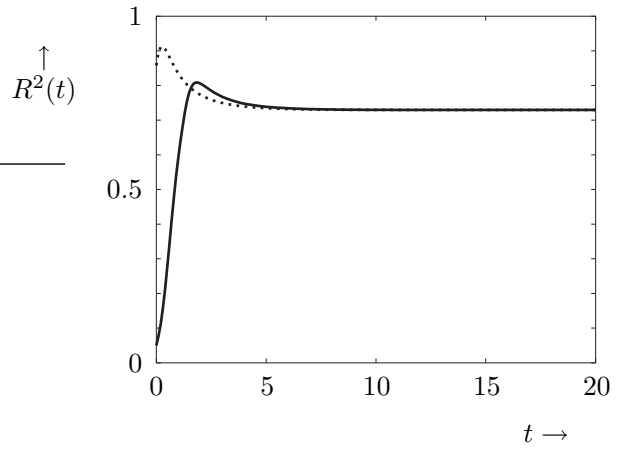

(b) $k=2.3$

Figure 7: Case $N=20$ : time evolution of the magnitude squared of the order parameter, $R^{2}(t)$, for two different initial condition (indicated by a dashed and solid line respectively), and two values of $k$. In the left panel, the value of $k(2.1)$ is slightly below the known upper bound on $k_{\mathrm{c}}(2.2281)$ and the system does not converge to a fixed point; in the right panel the value of $k(2.3)$ is slightly above the known upper bound on $k_{\mathrm{c}}$ and the system converges to a fixed point, as expected.

of the frequencies with their mean removed (Corollary 7 ). These bounds were shown to be the tightest possible, in the sense that we can find realizations of the intrinsic frequencies for which the upper bound is attained, and others for which the critical coupling is arbitrarily close to the lower bound. Finally, we proposed an efficient algorithm (Algorithm 1) for computing the critical coupling to within arbitrary bounds in a finite number of steps. In future work we shall seek to extend the present analysis to complex networks of arbitrary topology, and investigate more closely the impact of the shape of the distribution of intrinsic frequencies on the value of the critical coupling. We shall also consider the important question of stability, and present analytical results for the limit case when the number of oscillators tends to infinity.

\section{References}

[1] J. Acebrón, L. Bonilla, C. Pérez Vicente, F. Ritort, and R. Spigler. The kuramoto model: a simple paradigm for synchronization phenomena. Reviews of Modern Physics, 77:137-185, 2005.

[2] A. Beuter, L. Glass, M.C. Mackey, and M.S. Titcombe, editors. Nonlinear Dynamics in Physiology and Medicine. Springer, 2003.

[3] N. Chopra and M. Spong. On synchronization of kuramoto oscillators. In Proceedings of the 44th IEEE Conference on Decision and Control, Seville, Spain, pages 3916-3922, 2005. 


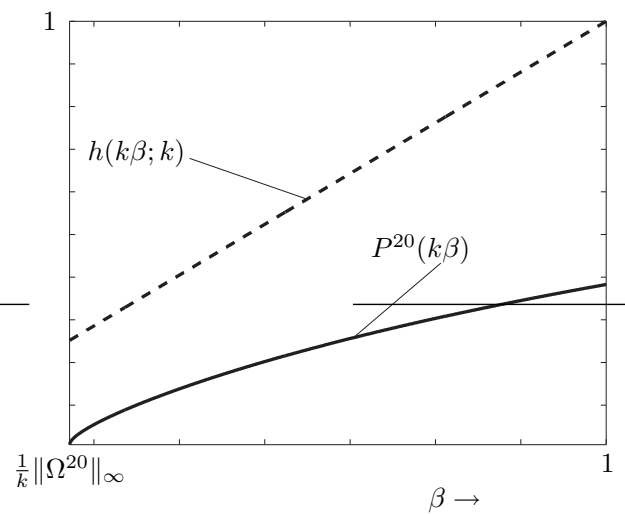

(a) $k=2.1$

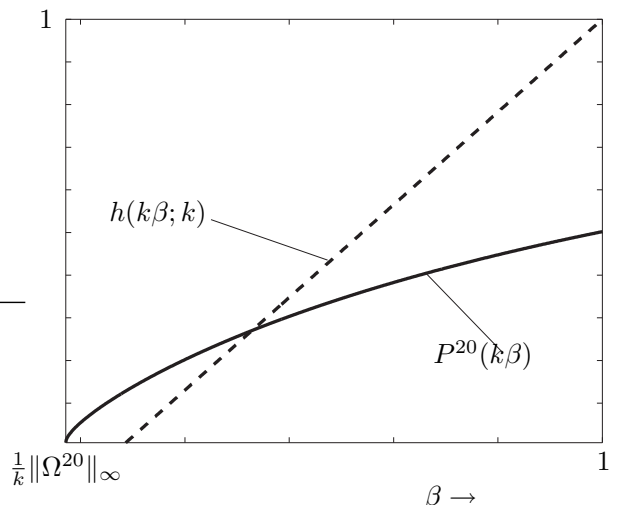

(b) $k=2.3$

Figure 8: Case $N=20$ : the graph of $P^{20}(k \beta)$ (Eqn. (71)) vs. $\beta$ for $k=2.1,2.3$ and $\beta \in\left[\frac{1}{k}\left\|\Omega^{20}\right\|_{\infty}, 1\right]$. The dashed line is the graph of $h(k \beta ; k)=\beta$ (Eqn. (72)). An intersection corresponds to a solution of the fixed point equation $P(k \beta)=$ $h(k \beta ; k)$, and thus, by Theorem 2, to a fixed point of the system (17).

[4] R. Diestel. Graph Theory. Springer-Verlag, 2000.

[5] L. Glass. Synchronization and rhythmic processes in physiology. Nature Review, 410:277-284, 2001.

[6] M. Golubitsky and Ian Stewart. Nonlinear Dynamics of Networks: the Groupoid Formalism. Bulletin of the American Mathematical Society, 43(3):305-364, 2006.

[7] H. Hong, M. Choi, and B. Kim. Synchronization on small-world networks. Physical Review E, 65(026139), 2002.

[8] H. Hong, H. Park, and M. Choi. Collective synchronization in spatially extended systems of coupled oscillators with random frequencies. Physical Review E, 72(036217), 2005.

[9] A. Jadbabaie, N. Motee, and M. Barahona. On the stability of the kuramoto model of coupled nonlinear oscillators. In Proceedings of the American Control Conference, Boston, USA, pages 4296 - 4301, 2004.

[10] Y. Kuramoto. Self-entrainment of a population of coupled nonlinear oscillators. In H. Araki, editor, International Symposium on Mathematical Problems in Theoretical Physics, volume 39 of Lecture Notes in Physics. Springer, 1975.

[11] Y. Kuramoto. Chemical Oscillations, Waves and Turbulence. Springer, 1984. 
[12] Y. Maistrenko, O. Popovych, and P. Tass. Desynchronization and Chaos in the Kuramoto Model, volume 671 of Lecture Notes in Physics. 2005.

[13] Y. Maistrenko, V. Popovych, O. Burylko, and P. Tass. Mechanism of Desynchronization in the Finite-Dimensional Kuramoto Model. Physical Review Letters, 93(8), 2004.

[14] Y. Maistrenko, V. Popovych, and P. Tass. Chaotic Attractor in the Kuramoto Model. International Journal of Bifurcation and Chaos, 15(11):3457-3466, 2005.

[15] Y. Moreno and A. Pacheco. Synchronization of kuramoto oscillators in scale-free networks. Europhysics Letters, 68:603-609, 2004.

[16] J.D. Murray. Mathematical Biology. Springer, 3rd edition, 2002.

[17] A. Pikovsky, M. Rosenblum, and J. Kurths. Synchronization: a universal concept in nonlinear sciences. Cambridge University Press, 2003.

[18] V. Popovych, Y. Maistrenko, and P. Tass. Phase chaos in coupled oscillators. Physical Review E, 71(065201), 2005.

[19] J. Rogge and D. Aeyels. Existence of partial entrainment and stability of phase locking behavior of coupled oscillators. Progress of Theoretical Physics, 112:921-942, 2004.

[20] J. Rogge and D. Aeyels. Stability of phase locking in a ring of unidirectionally coupled oscillators. Journal of Physics A, 37:11135-11148, 2004.

[21] R. Sepulchre. Oscillators as systems and synchrony as a design principle. In L. Menini, L. Zaccarian, and C. T. Abdallah, editors, Current trends in nonlinear systems and control, Lecture Notes in Control and Information Sciences. Birkhauser, 2006.

$[22]$ R. Sepulchre, D. Paley, and N.E. Leonard. Group coordination and cooperative control of steered particles in the plane. In K. Y. Pettersen, J. T. Gravdahl, and H. Nijmeijer, editors, Group Coordination and Cooperative control, Lecture Notes in Control and Information Sciences, pages 217-232. Spinger-Verlag, London, 2006.

[23] S. Strogatz. From kuramoto to crawford: exploring the onset of synchronization in populations of coupled oscillators. Physica D, 143:1-20, 2000.

[24] S. Strogatz. Exploring complex networks. Nature, 410:268 - 276, 2001.

[25] M. Verwoerd. Fixed-point analysis of a finite system of kuramoto oscillators. In Proceedings of the Fourth Irish Conference on the Mathematical Foundations of Computer Science and Information Technology (MFCSIT), Cork, Ireland, 2006. 
[26] M. Verwoerd and O. Mason. Conditions for the existence of fixed points in a finite system of kuramoto oscillators. In Proceedings of the American Control Conference, New York, NY, pages -, 2007.

[27] D. Watts and S. Strogatz. Collective dynamics of 'small-world' networks. Nature, 393(6684)(6684):440-2, 1998. 